\title{
Solution of the quantum fluid dynamical equations with radial basis function interpolation
}

\author{
Xu-Guang Hu, Tak-San Ho, and Herschel Rabitz \\ Department of Chemistry, Princeton University, Princeton, New Jersey 08544-1009 \\ Attila Askar \\ Department of Mathematics, Koç University, 80860 Istanbul, Turkey \\ (Received 8 December 1999)
}

\begin{abstract}
The paper proposes a numerical technique within the Lagrangian description for propagating the quantum fluid dynamical (QFD) equations in terms of the Madelung field variables $R$ and $S$, which are connected to the wave function via the transformation $\psi=\exp \{(R+i S) / \hbar\}$. The technique rests on the QFD equations depending only on the form, not the magnitude, of the probability density $\rho=|\psi|^{2}$ and on the structure of $R=\hbar / 2 \ln \rho$ generally being simpler and smoother than $\rho$. The spatially smooth functions $R$ and $S$ are especially suitable for multivariate radial basis function interpolation to enable the implementation of a robust numerical scheme. Examples of two-dimensional model systems show that the method rivals, in both efficiency and accuracy, the split-operator and Chebychev expansion methods. The results on a three-dimensional model system indicates that the present method is superior to the existing ones, especially, for its low storage requirement and its uniform accuracy. The advantage of the new algorithm is expected to increase for higher dimensional systems to provide a practical computational tool.
\end{abstract}

PACS number(s): 02.70.-c, 02.60.Ed, 03.65. $-\mathrm{w}$

\section{INTRODUCTION}

During the past decade, significant progress has been made in the time-dependent treatment of atomic and molecular dynamics and bound-state problems. A variety of methods has been proposed for numerically solving the timedependent Schrödinger equation [1-6]. A major consideration is the need to attain an accurate and efficient approximate representation to often highly oscillatory wave functions, particularly, in more than one dimension. Both the spectral and pseudospectral methods employ a tensor product representation as a means to treat multidimensional systems, despite this representation being neither efficient nor accurate as a numerical scheme due to the rapid increase in the total number of basis functions or quadrature points as the dimension rises.

The causal interpretation of quantum mechanics in the de Broglie-Bohm theory has attracted a great deal of interest [7-10] since Madelung, de Broglie, and Bohm's pioneering works [12-14]. With the wave function $\psi$ written in a polar form $\psi(\mathbf{r}, t)=\sqrt{\rho(\mathbf{r}, t)} e^{i S(\mathbf{r}, t) / \hbar}[12]$, the de Broglie-Bohm theory possesses an intuitive physical representation as quantum fluid dynamics (QFD), reminiscent of classical fluid dynamics. Besides its conceptual importance, the potential numerical advantage of the QFD formulation over working with the Schrödinger equation can be attributed to the oscillatory real and imaginary parts of the complex-valued wave function $\psi$ being replaced by the slowly varying density $\rho$ and phase $S$ over the configuration space. In classical fluid dynamics, the motion of fluid particles can be described in either Eulerian or Lagrangian descriptions [15] of the dynamics, respectively, either by fixing the "monitors" in space or by placing the "monitors" on the fluid particles. These two equivalent descriptions can also be employed in the QFD formulation. The QFD formulation as a timedependent approach has successfully been applied to treat several illustrations within both the Lagrangian [16-22] and the Eulerian [23-35] descriptions.

The QFD method based on the slowly varying density $\rho$ was implemented for studying the photodissociation of $\mathrm{NOCl}$ and $\mathrm{NO}_{2}$ within both the Lagrangian [20] and the Eulerian [35] descriptions. In this paper, we propose an efficient and accurate numerical technique for solving the QFD equations based on the slowly varying function $R=\hbar / 2 \ln \rho$ in conjunction with multivariate radial basis function (RBF) interpolation. To this end, a set of dynamical equations is derived and implemented in the Lagrangian description. In our previous papers [36], RBF's have been used to solve the bound-state Schrödinger equation. The most promising feature of the numerical scheme proposed in this paper is its potential for application to multidimensional systems.

The paper is organized as follows. Section II presents the central motivation and RBF multivariate interpolation procedure. Section III is devoted to the numerical implementation of the method, and Sec. IV presents several illustrations. Section $\mathrm{V}$ concludes the presentation.

\section{METHODOLOGY}

\section{A. Motivation}

For simplicity, consider a single particle of mass $\mu$ in a potential $V(\mathbf{r}, t)$. The QFD equations within the Eulerian description can be obtained by inserting the polar form of a complex wave function $\psi(\mathbf{r}, t)=\sqrt{\rho(\mathbf{r}, t)} e^{i S(\mathbf{r}, t) / \hbar}$ into the Schrödinger equation, separating the real and imaginary parts, and by defining $\mathbf{v}=\nabla S / \mu$ as the "velocity" of the particle. The result is a pair of coupled nonlinear partial differential equations

$$
\frac{\partial \rho}{\partial t}+\nabla \cdot \mathbf{J}=0
$$




$$
\mu\left(\frac{\partial}{\partial t}+\mathbf{v} \cdot \nabla\right) \mathbf{v}=-\nabla\left(V+V_{q}\right)=\mathbf{F},
$$

where $\rho=\rho(\mathbf{r}, t)=|\psi(\mathbf{r}, t)|^{2}$ is the probability density, $\mathbf{J}$ $=\mathbf{v} \rho$ the probability current density of the system, $\mathbf{F}=$ $-\nabla\left(V+V_{q}\right)$ the total force acting on the particle, and

$$
V_{q}=-\frac{\hbar^{2}}{2 \mu} \frac{\nabla^{2} \sqrt{\rho}}{\sqrt{\rho}},
$$

is the quantum potential carrying all the quantum effects of the system. Equation (2.1) is the quantum probability continuity equation and Eq. (2.2) is the quantum analog of the classical Newton equation upon identification of the total derivative as $d / d t=\partial / \partial t+\mathbf{v} \cdot \nabla$ and the total potential as $V_{\text {total }}=V+V_{q}$

In the Lagrangian description, the QFD equations are of the form

$$
\begin{gathered}
\frac{d}{d t} \rho(\mathbf{r}(t), t)+\rho(\mathbf{r}(t), t) \nabla \cdot \mathbf{v}(\mathbf{r}(t), t)=0 \\
\mu \frac{d}{d t} \mathbf{v}(\mathbf{r}(t), t)=-\nabla\left[V(\mathbf{r}(t), t)+V_{q}(\mathbf{r}(t), t)\right]=\mathbf{F}(\mathbf{r}(t), t),
\end{gathered}
$$

where the time dependence of the trajectory $\mathbf{r}(t)$ is explicit and the total derivative with respect to time has replaced the the partial derivative in the Eulerian description. Note that the evolution of the density $\rho$ in Eq. (2.4) can also be rearranged into the evolution of the quantity $\ln \rho$. In this case, the quantum potential can be further written in a general form as

$$
V_{q}=-\frac{\hbar^{2}}{4 \mu}\left\{\nabla^{2} \ln \rho+\frac{1}{2}[\nabla \ln \rho]^{2}\right\} .
$$

Equations (2.3) and (2.6) show that the quantum potential depends only on the form of the density rather than its magnitude $[9,10]$. At the heart of the $\rho$-based QFD formulation is the assumption that the density $\rho$ and phase $S$ (or $\mathbf{v}$ $=\nabla S / \mu)$ are more slowly varying functions than the generally oscillatory real and imaginary parts of the corresponding complex-valued wave function. The discussion above further suggests that the density $\rho$ can be replaced by the quantity $\ln \rho$ without altering the nature of the QFD equations. This replacement has several suggestive numerical advantages. First, $\ln \rho$ should be a more slowly varying function than its argument $\rho$, and working with the former should make the QFD method numerically even more expedient. Second, the practical dynamical range of $\ln \rho$ should be much smaller than that of $\rho$. For example, if $\rho$ changes in a truncated region $10^{-14} \leqslant \rho \leqslant 1$ (i.e., $10^{-14}$ can be considered as numerically zero in usual double precision computations), then $\ln \rho$ will only range accordingly from -32 to 0 . Third, the structure of $\ln \rho$ is in general simpler than that of $\rho$ itself. For example, the density of a compact Gaussian wave packet possesses the form $\exp \left\{-\alpha(\mathbf{r}-\mathbf{r}(t))^{2}\right\}$ in its spatial part while $\ln \rho$ is a quadratic polynomial. Starting with a compact Gaussian wave packet, the evolution of dynamics is a continuous deformation of the initial wave packet under the influence of the potential, and the logarithm of the deformed density at different instants of time can usually be approximated by proper polynomials. Consequently, the numerical advantage of treating $\ln \rho$ in QFD, instead of $\rho$, should be capable of greatly accelerating the solution while attaining high accuracy.

In contrast to the Schrödinger equation, Eqs. (2.2) and (2.5) in the QFD formulation deal with the force field $\mathbf{F}$ that contains a third-order spatial derivative of $\rho$ whose accurate evaluation becomes computationally difficult, especially, as the dimension of the system increases. To overcome this difficulty, note that the direct result of the polar transformation is the quantum Hamilton-Jacobian equation [8] [see Eqs. (2.7) or (2.10)] and the quantum probability continuity equation [see Eq. (2.1)]. The loss of part of the hydrodynamic analogy arising from using the Hamilton-Jacobi equation in place of its Newton counterpart is not of any concern numerically because of the equivalence between these two settings.

Writing $\psi$ in the Madelung form $\psi=\exp \{(R+i S) / \hbar\}$ and following the same procedure leading to Eqs. (2.1) and (2.2), an equivalent QFD formulation in the Eulerian description is arrived at

$$
\begin{gathered}
\frac{\partial S}{\partial t}+\frac{(\nabla S)^{2}}{2 \mu}+V+V_{q}=0, \\
\frac{\partial R}{\partial t}+\frac{1}{\mu}\left\{\nabla R \cdot \nabla S+\frac{\hbar}{2} \nabla^{2} S\right\}=0,
\end{gathered}
$$

where the quantum potential has a new form $V_{q}=$ $-1 / 2 \mu\left\{(\nabla R)^{2}+\hbar \nabla^{2} R\right\}$ with $2 R=\hbar \ln \rho$ [cf. Eq. (2.6)]. Alternatively, by taking the total derivatives of $R$ and $S$ with respect to time, i.e.,

$$
\begin{aligned}
& \frac{d R}{d t}=\frac{\partial R}{\partial t}+\mathbf{v} \cdot \nabla R \\
& \frac{d S}{d t}=\frac{\partial S}{\partial t}+\mathbf{v} \cdot \nabla S,
\end{aligned}
$$

the corresponding QFD equations in the Lagrangian description can be expressed as

$$
\begin{gathered}
\frac{d S(\mathbf{r}(t), t)}{d t}=\frac{[\nabla S(\mathbf{r}(t), t)]^{2}}{2 \mu}-V(\mathbf{r}(t), t)-V_{q}(\mathbf{r}(t), t) \\
\frac{d R(\mathbf{r}(t), t)}{d t}=-\frac{\hbar}{2 \mu} \nabla^{2} S(\mathbf{r}(t), t)
\end{gathered}
$$

These two equations are the new basis for numerical implementation of the present $R$-based QFD method. As in Eqs. (2.4) and (2.5), the explicit time dependence has appeared in $\mathbf{r}(t)$, which is completely determined through the relation

$$
\frac{d \mathbf{r}(t)}{d t}=\mathbf{v}(\mathbf{r}(t), t)=\frac{\nabla S(\mathbf{r}(t), t)}{\mu} .
$$




\section{B. Interpolation}

Function representation is of fundamental importance in numerically solving ordinary or partial differential equations [37]. The quality of an interpolation scheme is dictated by the basis functions defining the interpolator, the total number of interpolating points (i.e., grid points), and the distribution of these points. In the Lagrangian description, the grid points of the system instantaneously match the deformation of the wave packet during the evolution. One main advantage of the Lagrangian picture is that at each instant of time the interpolation only needs to be carried out in a limited region in which the magnitude of the wave packet is significant. This keeps the total number of grid points relatively small even in high dimension while still maintaining high accuracy. The disadvantage is that grid points inevitably become scattered, and almost all sophisticated regular grid based methods, such as finite difference, discrete variable representation (DVR) [38], Fourier pseudospectral approach [39,40], and distributed approximating functionals (DAF) [41], are inappropriate in this circumstance.

Recently, due to its simplicity and accuracy, radial basis function $(\mathrm{RBF})$ interpolation has attracted considerable interest for interpolating multivariate scattered data $[42,43]$. A $\operatorname{RBF} \phi(\|\mathbf{r}\|)$ is a function that depends only on the distance $\|\mathbf{r}\|$, with $\|\cdot\|$ denoting the norm, and it maps a $D$-dimensional quantity $\mathbf{r}$ in the real vector space $\mathfrak{R}^{D}$ to a one-dimensional quantity $\phi$ in the real number space $\mathfrak{R}^{1}$. In general, the RBF interpolation problem can be posed as follows. Consider an arbitrary set of $N$ distinct scattered points $X=\left\{\mathbf{r}_{1}, \mathbf{r}_{2}, \ldots, \mathbf{r}_{N}\right\}$ in $\mathfrak{R}^{D}$ and the corresponding scattered data $F=\left\{f\left(\mathbf{r}_{1}\right), f\left(\mathbf{r}_{2}\right), \ldots, f\left(\mathbf{r}_{N}\right)\right\}$ for a function $f(\mathbf{r})$, the task of RBF interpolation is to find an approximation $L_{f}(\mathbf{r})$ to $f$ of the form

$$
L_{f}(\mathbf{r})=\sum_{i=1}^{N} c_{i} \phi\left(\left\|\mathbf{r}-\mathbf{r}_{i}\right\|\right),
$$

where the real coefficients $c_{1}, c_{2}, \ldots, c_{N}$ solve the linear system

$$
f\left(\mathbf{r}_{j}\right)=\sum_{i=1}^{N} c_{i} \phi\left(\left\|\mathbf{r}_{j}-\mathbf{r}_{i}\right\|\right), \quad j=1,2, \ldots, N
$$

with provision that the symmetric interpolation matrix $\mathbf{A}$ $=\left[A_{i j}\right]=\left[\phi\left(\left\|\mathbf{r}_{j}-\mathbf{r}_{i}\right\|\right)\right]$ be nonsingular. It has been shown that as long as the RBF $\phi\left(\left\|\mathbf{r}-\mathbf{r}^{\prime}\right\|\right)$ is conditionally positive definite in the sense that, for any set of $N$ complex numbers $\left\{a_{i}\right\}_{i=1}^{N}$,

$$
\sum_{i, j=1}^{N} a_{j}^{*} \phi\left(\left\|\mathbf{r}_{j}-\mathbf{r}_{i}\right\|\right) a_{i} \geqslant 0
$$

then the interpolation matrix $\mathbf{A}$ is always nonsingular and thus Eq. (2.14) is solvable [44].

The accuracy and convergence of different RBF's [cf. Eq. (2.16)] in multivariate approximation has been carefully investigated $[42,43]$. One of the most important theoretical findings regarding $\mathrm{RBF}$ interpolation is that each positivedefinite RBF can generate a reproducing kernel Hilbert space (RKHS) H, endowed with a proper inner product. As a result, any well-behaved function can be optimally recovered within $\mathbf{H}$ from a set of scattered data by the corresponding RBF interpolation [42-46]. To the end, based on the RKHS theory [47] and the RBF approximation theory described above, an approach has been developed to numerically solve the multidimensional bound-state Schrödinger equation [36]. The RBF interpolation is also attractive for analytical construction of potential energy surfaces using $a b$ initio data [48]. The following list covers several RBF's that have been extensively studied in function approximation theory [49]:

$$
\begin{aligned}
& \phi(r)=(-1)^{m}\left(c^{2}+r^{2}\right)^{\beta / 2}(2 m-2<\beta<2 m) \quad \text { multiquadrics } \\
& \phi(r)=\left(c^{2}+r^{2}\right)^{-\beta / 2}(\beta>0) \\
& \phi(r)=(-1)^{m} r^{2 m-2} \ln (r) \\
& \phi(r)=(-1)^{m}\left(c^{2}+r^{2}\right)^{m-1} \ln \left(c^{2}+r^{2}\right)^{1 / 2} \\
& \phi(r)=\frac{2 \pi^{n / 2}}{\Gamma(k)} K_{k-n / 2}(r)\left(\frac{r}{2}\right)^{k-n / 2}(2 k>n) \quad \text { Sobolev splines } \\
& \phi(r)=e^{-(c r)^{2}} \\
& \text { inverse multiquadrics } \\
& \text { thin-plate splines } \\
& \text { shifted thin-plate splines } \\
& \text { Gaussian, }
\end{aligned}
$$

where $r=\|\mathbf{r}\|=\sqrt{\sum_{i=1}^{D} x_{i}^{2}}$ is the radial distance in the $D$-dimensional Euclidean space, and $m$ is usually chosen in such a way that it coincides with the order of polynomials to be included in the interpolation space. Among them, the multiquadrics with $\beta=1$ has recently been explored in computational fluid dynamics [50], while the Gaussian has had a long history of applications in solving molecular bound-state problems [51-55]. More recently, the inverse multiquadrics and Sobolev splines have also been exploited for bound-state problems [36]. In this paper, the multiquadrics and the thinplate splines will be employed for approximating the QFD variables $R$ and $S$ in the Lagrangian description Eqs. (2.10) and (2.11).

\section{Perspectives}

Several interrelated issues are pertinent to the numerical implementation of the QFD formulation, especially, in its 
Lagrangian description in Eqs. (2.10) and (2.11): (i) the initial state of the wave function, (ii) the nodes of the wave function, and (iii) the phase of the wave function. These points are addressed below.

First, the use of a single compact node-free initial wave packet should be generally sufficient to extract all relevant dynamical information, as such a compact wave packet will be a superposition of nearly all the quantum states of the dynamical system. In certain cases different symmetryadapted initial states may be used [56] to avoid the simultaneous excitation of closely spaced energy levels and thus to improve the numerical resolution and accuracy of calculated degenerate energy levels. This paper deals only with compact node-free Gaussian initial wave packets.

Second, the trajectory of the particle obeying Eq. (2.5) will never pass through a point $\rho(\mathbf{r}(t), t)=0$ as long as $\rho(\mathbf{r}(0), 0) \neq 0$ and $\nabla \cdot \mathbf{v}(\mathbf{r}(0), 0)$ is finite [9]. This can be readily understood by writing Eq. (2.4) in terms of its integral equivalence as follows

$$
\rho(\mathbf{r}(t), t)=\rho(\mathbf{r}(0), 0) \exp \left\{-\int_{0}^{t} d t^{\prime} \nabla \cdot \mathbf{v}\left(\mathbf{r}\left(t^{\prime}\right), t^{\prime}\right)\right\},
$$

indicating that the associated density $\rho(\mathbf{r}(t), t)$ will never become zero during the evolution. As a result, the quantity $R(\mathbf{r}(t), t)$ will remain finite and the corresponding quantum potential Eq. (2.6) can be evaluated without numerical difficulty throughout the propagation within the Lagrangian QFD framework.

Third, the multivalued nature of $S$ does not cause the collapse of the QFD equations. Here, the QFD equations only involve both the time derivative and the gradient of $S$, i.e., $d S / d t$ and $\mathbf{v}=\nabla S / \mu$, which are single-valued functions of position and time. This means that at each space-time point there is a unique tangent vector associated with $\nabla S$, and consequently, only one trajectory passes through that point at each instant of time. This is consistent with the quantum probability continuity equation, which maps the non-nodal point regions into one another along the trajectories.

A detailed discussion of the node and phase issues can be found in [9]. Moreover, it has been shown that for a large class of potentials and typical initial wave functions, the global solution of the QFD equations exists and is unique [11]. In conclusion, no serious difficulty is expected in the numerical solution of Eqs. (2.10)-(2.12). One important feature arising from working with $R$ and $S$ in the Lagrangian description is that, starting with an initial wave packet without a node, i.e., $R(\mathbf{r}(0), 0) \neq-\infty$, then subsequently nowhere will $R$ become singular.

\section{NUMERICAL DETAILS}

\section{A. Time propagation}

In a previous paper [20], the explicit central differencing scheme was adopted for time integration of the QFD equations. In the case of the Schrödinger equation, this scheme has been shown to be stable under the condition $\left(\Delta t / 2 \mu \Delta x^{2}\right)<1$, where $\Delta t$ and $\Delta x$ are the discretized temporal and spatial grid sizes [57]. In the present case, working in the Lagrangian description, the new stability condition $v \Delta t / \Delta x<1$ is established in terms of the particle's motion, where $v$ is the velocity of a particle and $\Delta x$ is the shortest distance among all the moving grids [20]. A second-order time propagation scheme for Eqs. (2.10) and (2.11) can be implemented as follows:

$$
\begin{gathered}
S(t+\Delta t)=S(t-\Delta t)+\Delta t\left\{\frac{[\nabla S(t)]^{2}}{\mu}-2\left[V(t)+V_{q}(t)\right]\right\} \\
R(t+\Delta t)=R(t-\Delta t)-\frac{\hbar \Delta t}{\mu} \nabla^{2} S(t)
\end{gathered}
$$

where the $\mathbf{r}(t)$ dependence in $S$ and $R$ has been dropped for notational simplicity and the evolution of the particle's position is advanced according to its velocity as follows

$$
\mathbf{r}(t+\Delta t)=\mathbf{r}(t-\Delta t)+\frac{2 \Delta t}{\mu} \nabla S(t) .
$$

\section{B. Spatial representation}

In contrast to fixed grid methods, the construction of a multidimensional subspace on a mesh using RBF's distributed on irregular grid points is technically straightforward. Specifically, the interpolation (2.13) is formally independent of the dimensionality of the problem involved and all connections among grid points are properly done through the interpolation condition (2.14). The largest cost of RBF interpolation is the direct solution of the linear algebraic equation (2.14), which scales as the cube of total number $N$ of grid points. Nevertheless, this problem can easily be overcome by using the compactly supported RBF's as explained below.

In principle, any grid distribution for the spatial discretization of the QFD equations is acceptable as long as the underlying RBF interpolation can adequately sample the wave packet. Following the discussions in Secs. I and II, the QFD solutions can be implemented in three steps.

1. Initial preparation. (a) Choose an appropriate initial wave packet for the system under study; (b) set a density cutoff for the initial wave packet, e.g., $10^{-7}$, etc., depending on the required computational accuracy; (c) place grid points in terms of the structure of $R$ (or $\rho$ ) within the region determined by the initial density cutoff.

2. Spatial derivatives. The gradient and Laplacian are taken by directly (i.e., analytically) applying them to the interpolated $R(t)$ and $S(t)$ functions, respectively, evaluated on the dynamic grids $\left\{\mathbf{r}_{i}(t)\right\}$ at each instant of time $t$.

3. Propagation. With the chosen grid, the discretized field variables $R, S$ and the position $\mathbf{r}$ are propagated according to

$S_{i}(t+\Delta t)=S_{i}(t-\Delta t)+\Delta t\left\{\frac{\left[\nabla S_{i}(t)\right]^{2}}{\mu}-2\left[V_{i}(t)+V_{q i}(t)\right]\right\}$,

$$
\begin{gathered}
R_{i}(t+\Delta t)=R_{i}(t-\Delta t)-\frac{\hbar \Delta t}{\mu} \nabla^{2} S_{i}(t), \\
\mathbf{r}_{i}(t+\Delta t)=\mathbf{r}_{i}(t-\Delta t)+\frac{2 \Delta t}{\mu} \nabla S_{i}(t),
\end{gathered}
$$


where the index $i$ denotes the $i$ th grid point $\mathbf{r}_{i}$ at three different adjacent instants $\mathbf{r}_{i}(t+\Delta t), \mathbf{r}_{i}(t-\Delta t)$, and $\mathbf{r}_{i}(t)$ in each propagation cycle. Interpolation of $R(t)$ and $S(t)$ is performed at each instant of time $t$ by use of the RBF's distributed on the dynamic grids $\left\{\mathbf{r}_{i}(t)\right\}$ whose configuration changes in response to the action of the potential.

The RBF interpolation is a global scheme, however, in practice, compactly supported RBF's can be invoked to avoid solving a large linear algebraic system (2.14). A simple way to obtain the compactly supported RBF's is to introduce a suitable weight function. For example, suppose $\Omega$ is a hypersphere centered at $\mathbf{r}_{0}$ in a multidimensional space and the compactly supported weight function $w(\mathbf{r}$ $-\mathbf{r}_{0}$ ) satisfies

$$
w\left(\mathbf{r}-\mathbf{r}_{0}\right)= \begin{cases}1, & \text { if } \mathbf{r} \text { inside } \Omega \\ 0, & \text { if } \mathbf{r} \text { outside } \Omega,\end{cases}
$$

then the corresponding compactly supported RBF's are given as $\phi(\mathbf{r}) w\left(\mathbf{r}-\mathbf{r}_{0}\right)$. Systematically relocating $\Omega$ will cover the entire region of interest and all discretized quantities will be described locally through the RBF interpolation. It is apparent that at each position $\mathbf{r}_{0}$ only a small portion of the grid points reside in $\Omega$ and, thus, only a small linear algebraic system needs to be solved. In general, the number of grid points inside $\Omega$ at different positions $\mathbf{r}_{0}$ is not fixed for a given size of the hypershere because of the irregularity of the grid. Consequently, it may happen that only a few grid points are inside $\Omega$ in some positions and a great deal in others. In practice, the size of $\Omega$ may be distinct at each position $\mathbf{r}_{0}$ so that a sufficient number of grid points is always included to attain the required accuracy.

In this paper, a practical procedure to achieve this adaptable compactly supported interpolation scheme is introduced as follows.

1. All the $N$ grid points are initially numbered in an arbitrary manner.

2. A lower bound $r_{\text {min }}$ is set for the distance between any two grid points.

3. Upon choosing a grid point as a center, then a search is made for its $n-1$ closest neighbors under the conditions 1 $\ll n \ll N$ and $r_{i j}>r_{\text {min }}$, where $r_{i j}$ is the distance between grid points $i$ and $j$.

4. Local interpolation of $R$ and $S$ is performed over these $n$ points by solving the corresponding $n$ linear algebraic equations (2.14) based on the compactly supported RBF's.

5. Derivatives of $R$ and $S$ at these $n$ points are directly taken over the locally interpolated $R$ and $S$.

6. The results in steps 4 and 5 as well as their numbered indices are kept except for $n_{b}$ boundary grid points of each local interpolation.

7. Steps $1-6$ are repeated $N_{n}\left(N / n<N_{n}<N\right)$ times until all discretized quantities on the $N$ grid points are calculated.

The lower bound $r_{\min }$ in step 2 is set to prevent the linear algebraic system in step 4 from becoming ill-conditioned and the operations of step 5 eliminate any interpolation edge effects. It can happen that some grid points are shared by a couple of partly overlapped local interpolations in step 7 and thus the results in step 5 corresponding to these grid points are calculated many times. In this case, if these grid points are not located at the boundary of each local interpolation, the calculated results are kept when the corresponding grid points are first employed in a local interpolation. The proposed procedure usually implies a deformable $\Omega$ whose shape changes (i.e., the choices in step 3 generally do not prescribe a spherical domain) with the distribution of grid points in each local interpolation.

\section{NUMERICAL ILLUSTRATIONS}

Numerical tests in this section are performed on twodimensional (2D) and (3D) free particle models, a 2D coherent state model and a 2D model of $\mathrm{NOCl}$ photodissociation. For comparison, parallel calculations are also carried out using the Chebychev expansion (CE) [3] and split-operator (SO) [56] methods, respectively. It is known that the CE method is not efficient for the short-time steps although some methods are available $[1,3]$ to enhance its efficiency. Therefore, to assess the efficiency of the QFD method, one single giant time step in the CE method to complete the propagation is taken as a reference except for the $2 \mathrm{D}$ coherent state case that does not favor a giant time step in the CE method. Moreover, the same time steps are taken for the second-order SO and the QFD methods. Finally, due to the short-time feature of the QFD method, intermediate results are available for any quantity of interest requiring time integration. Atomic units are used throughout the calculations.

\section{A. Analytical test solutions and initial wave packets}

For a $D$-dimensional free particle with an initial momentum distribution chosen as a Gaussian in each dimension, the time-dependent solution of the Schrödinger equation can be written, in terms of $R$ and $S$ as

$$
\begin{aligned}
R\left(\left\{x_{i}\right\}, t\right)= & -\frac{1}{2} \sum_{i=1}^{D}\left\{\frac{1}{2} \ln \left(\pi\left[\Delta x_{i}^{2}+\left(\frac{t}{\Delta x_{i} \mu_{i}}\right)^{2}\right]\right)\right. \\
& +\frac{\left(x_{i}-x_{0 i}-v_{0 i} t\right)^{2}}{\left.\Delta x_{i}^{2}+\left(\frac{t}{\Delta x_{i} \mu_{i}}\right)^{2}\right\},} \\
S\left(\left\{x_{i}\right\}, t\right)=\sum_{i=1}^{D}\left\{p_{0 i}\left(x_{i}-x_{0 i}\right)+\theta_{i}(t)\right. & \\
+ & \left.\frac{t}{2 \mu_{i}}\left[\frac{\left(x_{i}-x_{0 i}-v_{0 i} t\right)^{2}}{\Delta x_{i}^{2}\left[\Delta x_{i}^{2}+\left(\frac{t}{\Delta x_{i} \mu_{i}}\right)^{2}\right]}-p_{0 i}^{2}\right]\right\},
\end{aligned}
$$

where

$$
\theta_{i}(t)=\frac{1}{2} \tan \left(-\frac{t}{\Delta x_{i}^{2} \mu_{i}}\right),
$$


and $\mu_{i}, v_{0 i}, p_{0 i}, x_{0 i}$, and $\Delta x_{i}$ are the mass, initial velocity, momentum, position, and position width along each dimension, respectively.

For a $D$-dimensional harmonic oscillator, the timedependent coherent state is given by [8]

$$
\begin{gathered}
R(\mathbf{r}, t)=\frac{D}{4} \ln \left(\frac{\mu \omega}{\pi}\right)-\frac{\mu \omega}{2}\left(\mathbf{r}-\mathbf{r}_{c}(t)\right)^{2}, \\
S(\mathbf{r}, t)=\mathbf{p}_{c}(t)\left(\mathbf{r}-\mathbf{r}_{c}(t)\right)-\frac{1}{2} D \omega t,
\end{gathered}
$$

where

$$
\begin{gathered}
\mathbf{r}_{c}(t)=\mathbf{r}_{c}(0) \cos (\omega t)+(\mu \omega)^{-1} \mathbf{p}_{c}(0) \sin (\omega t), \\
\mathbf{p}_{c}(t)=-\mu \omega \mathbf{r}_{c}(0) \sin (\omega t)+\mathbf{p}_{c}(0) \cos (\omega t),
\end{gathered}
$$

with $\mathbf{r}_{c}(t)$ and $\mathbf{p}_{c}(t)$, respectively, being the position of the center of the coherent state and its conjugate momentum; $\mu$ and $\omega$ are, respectively, the mass and frequency.

For the simplified 2D model of $\mathrm{NOCl}$ photodissociation [58-60], the initial wave packet is a Gaussian [cf. Eqs. (4.1) and (4.2) at $t=0]$ with appropriate parameters.

\section{B. $\mathrm{NOCl}$}

For the NOCl molecule, the Hamiltonian based on Jacobi coordinates can be written as

$$
\begin{aligned}
\hat{H}= & -\frac{1}{2 \mu_{d} r_{d}} \frac{\partial^{2}}{\partial r_{d}^{2}} R-\frac{1}{2 \mu_{v} r_{v}} \frac{\partial^{2}}{\partial r_{v}^{2}} r_{v}-\frac{1}{2 I_{\theta} \sin \theta} \frac{\partial}{\partial \theta} \sin \theta \frac{\partial}{\partial \theta} \\
& +V\left(r_{d}, r_{v}, \theta\right),
\end{aligned}
$$

where $r_{v}$ is the bond length (vibrational coordinate) of fragment NO and $r_{d}$ the distance (dissociative coordinate) from $\mathrm{Cl}$ to the center the mass of fragment NO; $\theta$ indicates the angle between $r_{d}$ and $r_{v}$. The reduced masses $\mu_{d}, \mu_{v}$ and the moment of inertia $I_{\theta}$ for $\mathrm{NOCl}$ molecule are

$$
\begin{gathered}
\frac{1}{\mu_{d}}=\frac{1}{m_{\mathrm{N}}+m_{\mathrm{O}}}+\frac{1}{m_{\mathrm{Cl}}}, \frac{1}{\mu_{v}}=\frac{1}{m_{\mathrm{N}}}+\frac{1}{m_{\mathrm{O}}}, \\
\frac{1}{I_{\theta}}=\frac{1}{\mu_{v} r_{v}^{2}}+\frac{1}{\mu_{d} r_{d}^{2}},
\end{gathered}
$$

with $m_{\mathrm{N}}, m_{\mathrm{O}}$, and $m_{\mathrm{Cl}}$ being the masses of atoms $\mathrm{N}, \mathrm{O}$, and $\mathrm{Cl}$, respectively. Model potential energy surfaces $V\left(r_{d}, r_{v}, \theta\right)$ for the $S_{0}$ and $S_{1}$ states of NOCl are available [58-60]. For simplicity, the calculations were carried out with the angle variable $\theta$ fixed at a specific value. In this case, all parts involving angular derivatives in Eq. (4.8) disappear and the Hamiltonian reduces to a simple form

$$
\hat{H}=-\frac{1}{2 \mu}\left(\frac{\partial^{2}}{\partial x^{2}}+\frac{\partial^{2}}{\partial y^{2}}\right)+V\left(x, y, \theta_{0}\right) \text {, }
$$

where $\theta_{0}$ is frozen at $127.4^{\circ}$, the equilibrium value for the excited state $S_{1}, \mu=\sqrt{m_{d} m_{v}}$, and

$$
\begin{gathered}
x=\left(m_{d} / m_{v}\right)^{1 / 4} r_{d}, \\
y=\left(m_{v} / m_{d}\right)^{1 / 4} r_{v}, \\
\phi(x, y)=r_{d} r_{v} \psi\left(r_{d}, r_{v}, \theta_{0}\right),
\end{gathered}
$$

that $0 \leqslant x, y<\infty$ in Eq. (4.11).

\section{Results and discussion}

In fixed grid methods for solving the time-dependent Schrödinger equation, an adequate choice of spatial region to cover the wave packet propagation is important. Insufficient spatial coverage will cause unphysical boundary reflection that contaminates the wave packet. Too large a spatial domain can result in either inefficiency (i.e., the need for many grid points to maintain accuracy) or inaccuracy (i.e., when fewer than necessary grid points are used in an attempt to increase efficiency). These two considerations have been taken into account in our calculations using the CE and SO methods. The spatial regions and the numbers of grid points for each case discussed later in Table II have been chosen to yield the best results on balance in terms of accuracy and efficiency for these two methods. It is found that in twodimensional cases the fast Fourier transform (FFT) spatial representation incorporated in the $\mathrm{CE}$ and $\mathrm{SO}$ methods is both efficient and accurate, whereas, in three and higher dimensions the FFT representation becomes inefficient due to its tensor product form [40]. Although an optimal sampling for multidimensional systems may alleviate the latter grid size issue, its actual implementation can be extremely cumbersome [40]. As a result, the calculations for the 3D free particle in this paper were carried out on a supercomputer (CRAY J90). All other calculations were done on Sun workstation OS 5.6.

The RBF's used in the calculations for the QFD method are the multiquadrics and shifted-thin-plate spline [see Eq. (2.16)]. Due to the fact that the spatial dependence of $R$ and $S$ for the free particle and coherent state cases are at most quadratic, the parameters $\beta=3$ and $m=2$ are chosen for these two RBF's. For the 2D NOCl photodissociation calculation, the corresponding parameters for the RBF's were investigated; it is found that the parameters are only slightly dependent on the choice of $r_{\text {min }}$, the lower bound of the spacings set for preventing the local linear algebraic systems from becoming ill conditioned. Numerical tests have been carried out for $\beta=1,3,5,7,9$ and $m=1,2,3,4,5$ with good results found at $\beta=5,7$ and $m=3,4$. Insensitivity is found for the shifted parameter $c$ characterizing these two RBF's and its value can range from 15 to 35 (in atomic units) without altering the propagation results. Hereafter, all subsequent discussions for the 2D NOCl model involved $\beta=5, m=3$, and $c=20$. The multiquadrics and shifted-thin-plate spline, used in all model calculations, give results of almost the same accuracy.

Table I lists the number of grid points $n$ and boundary points $n_{b}$ employed in the local interpolation as well as the wave packet parameters for the different cases involved. Because of the simple structures of $R$ and $S$ in the free particle and coherent state cases, the number of grid points $n$ used in the local interpolation is taken to be the same as the total grid number $N$ listed in Table II. Tables II and III clearly show 
TABLE I. Parameters for wave packets and local interpolation (a.u.).

\begin{tabular}{lcc}
\hline \hline Free particle & $\begin{array}{c}\text { Coherent state } \\
\text { wave packet }\end{array}$ & NOCl model \\
\hline$\mu=5000$ & $\mu=5000$ & $\mu=5000$ \\
$\Delta x_{1}=\Delta x_{2}=\Delta x_{3}=0.25$ & $\omega=0.0032$ & $\Delta x=0.135, \Delta y=0.078$ \\
$x_{01}=x_{02}=x_{03}=0$ & $x_{1 c}(0)=x_{2 c}(0)=0$ & $x_{0} 4.314, y_{0}=2.155$ \\
& Local interpolations $\left.n, n_{b}\right)^{\mathrm{a}}$ & \\
\hline$(57,0)$ & $(25,0)$ & $\beta=5, m=3$ \\
$\beta=5, m=2$ & $\beta=5, m=2$ & $c=15 \sim 35$ \\
$c=15 \sim 35$ & $c=15 \sim 35$ & $(25,9)$ \\
\hline \hline
\end{tabular}

$\overline{{ }^{a} n}$ and $n_{b}$ are the numbers of grid points and boundary grid points used in each local interpolation, respectively.

that, compared to the CE and SO methods, the QFD technique requires substantially less computer overhead (memory and CPU time), especially in the three-dimensional case.

In the cases of the $2 \mathrm{D}$ free particle and the $2 \mathrm{D} \mathrm{NOCl}$ photodissociation, the storage requirement for the QFD method scales approximately as $25 \times 25=625$, which is the size of the local interpolation matrix in Eq. (2.14), while the storage needed for FFT used in the CE and SO methods scales as $2 \times 128^{2}=32768$, being $\sim 50$ times as large as that required by the QFD method. As the spatial dimension increases, the storage savings by the QFD method becomes very significant as seen in the $3 \mathrm{D}$ free particle case where the storage scaling for FFT rises to $2 \times 128^{3}$, while that of the

TABLE II. Data used for comparison calculations (a.u.).

\begin{tabular}{|c|c|c|c|c|c|c|c|c|}
\hline \multirow[t]{2}{*}{$\mathrm{PT}^{\mathrm{a}}$} & \multicolumn{3}{|c|}{$\mathrm{TS}^{\mathrm{b}}$} & \multicolumn{2}{|c|}{ TNGP $^{\mathrm{c}}$} & \multicolumn{2}{|c|}{$\mathrm{SS}^{\mathrm{d}}$} & \multirow{2}{*}{$\frac{\mathrm{GR}^{\mathrm{e}}}{\mathrm{CE} \& \mathrm{SO}}$} \\
\hline & $C E^{f}$ & $\mathrm{SO}^{\mathrm{g}}$ & QFD & $\mathrm{CE} \& \mathrm{SO}$ & QFD & $\mathrm{CE} \& \mathrm{SO}$ & QFD & \\
\hline \multicolumn{9}{|c|}{ 2D free particle case } \\
\hline 1000 & 1000 & 5 & 5 & $128^{2}$ & 25 & $2 \times 128^{2}$ & $25^{2}$ & {$[-5,7]^{2}$} \\
\hline 2000 & 2000 & 5 & 5 & $128^{2}$ & 25 & $2 \times 128^{2}$ & $25^{2}$ & {$[-7,10]^{2}$} \\
\hline 3000 & 3000 & 5 & 5 & $128^{2}$ & 25 & $2 \times 128^{2}$ & $25^{2}$ & {$[-10,13]^{2}$} \\
\hline 4000 & 4000 & 5 & 5 & $128^{2}$ & 25 & $2 \times 128^{2}$ & $25^{2}$ & {$[-13,17]^{2}$} \\
\hline \multicolumn{9}{|c|}{ 3D free particle case } \\
\hline 1000 & 1000 & 5 & 5 & $128^{3}$ & 57 & $2 \times 128^{3}$ & $57^{2}$ & {$[-5,7]^{3}$} \\
\hline 2000 & 2000 & 5 & 5 & $128^{3}$ & 57 & $2 \times 128^{3}$ & $57^{2}$ & {$[-7,10]^{3}$} \\
\hline 3000 & 3000 & 5 & 5 & $128^{3}$ & 57 & $2 \times 128^{3}$ & $57^{2}$ & {$[-10,13]^{3}$} \\
\hline 4000 & 4000 & 5 & 5 & $128^{3}$ & 57 & $2 \times 128^{3}$ & $57^{2}$ & {$[-13,17]^{3}$} \\
\hline \multicolumn{9}{|c|}{ 2D coherent state case } \\
\hline 1000 & 1 & 1 & 1 & $32^{2}$ & 25 & $2 \times 32^{2}$ & $25^{2}$ & {$[-2,2]^{2}$} \\
\hline 2000 & 1 & 1 & 1 & $32^{2}$ & 25 & $2 \times 32^{2}$ & $25^{2}$ & {$[-2,2]^{2}$} \\
\hline 3000 & 1 & 1 & 1 & $32^{2}$ & 25 & $2 \times 32^{2}$ & $25^{2}$ & {$[-2,2]^{2}$} \\
\hline 4000 & 1 & 1 & 1 & $32^{2}$ & 25 & $2 \times 32^{2}$ & $25^{2}$ & {$[-2,2]^{2}$} \\
\hline \multicolumn{9}{|c|}{ 2D model for $\mathrm{NOCl}$} \\
\hline 500 & 500 & 1.25 & 1.25 & $256 \times 32$ & 127 & $2 \times 256 \times 32$ & $25^{2}$ & {$[1.8,2.8][3.5,8]$} \\
\hline 1000 & 1000 & 1.25 & 1.25 & $256 \times 32$ & 127 & $2 \times 256 \times 32$ & $25^{2}$ & {$[1.8,2.8][3.5,8]$} \\
\hline 1500 & 1500 & 1.25 & 1.25 & $256 \times 32$ & 127 & $2 \times 256 \times 32$ & $25^{2}$ & {$[1.8,2.8][3.5,8]$} \\
\hline
\end{tabular}

${ }^{\mathrm{a}} \mathrm{PT}=$ propagation time.

${ }^{\mathrm{b}} \mathrm{TS}=$ time step.

${ }^{\mathrm{c}} \mathrm{TNGP}=$ total number of grid points.

${ }^{\mathrm{d}} \mathrm{SRS}=$ storage scaling.

${ }^{\mathrm{e}} \mathrm{GR}=$ grid region.

${ }^{\mathrm{f}} \mathrm{CE}=$ Chebychev expansion.

${ }^{\mathrm{g}} \mathrm{SO}=$ split operator. 
TABLE III. Comparison of accuracy and efficiency.

\begin{tabular}{|c|c|c|c|c|c|c|}
\hline \multirow[t]{2}{*}{ Propagation time (a.u.) } & \multicolumn{3}{|c|}{ CPUT $(\mathrm{sec})^{\mathrm{a}}$} & \multicolumn{3}{|c|}{ Error $^{b}$} \\
\hline & $\mathrm{CE}$ & $\mathrm{SO}$ & QFD & $\mathrm{CE}$ & $\mathrm{SO}$ & QFD \\
\hline \multicolumn{7}{|c|}{ 2D free particle case ${ }^{c}$} \\
\hline 1000 & 19 & 40 & 1 & $3.1(-14)$ & $3.1(-14)$ & $3.2(-6)$ \\
\hline 2000 & 18 & 79 & 2 & $8.3(-7)$ & $8.3(-7)$ & $2.0(-6)$ \\
\hline 3000 & 16 & 125 & 4 & $6.3(-4)$ & $6.3(-4)$ & $1.8(-6)$ \\
\hline 4000 & 13 & 165 & 6 & $1.8(-3)$ & $1.8(-3)$ & $3.0(-6)$ \\
\hline \multicolumn{7}{|c|}{$3 D$ free particle case ${ }^{d}$} \\
\hline 1000 & 16346 & & 57 & $1.8(-14)$ & & $2.5(-6)$ \\
\hline 2000 & 15742 & & 124 & $4.2(-7)$ & & $1.5(-6)$ \\
\hline 3000 & 12144 & & 194 & $1.3(-4)$ & & $2.3(-6)$ \\
\hline 4000 & 10098 & & 268 & $3.6(-3)$ & & $3.5(-6)$ \\
\hline \multicolumn{7}{|c|}{$2 \mathrm{D}$ coherent state case $^{\mathrm{c}}$} \\
\hline 1000 & 49 & 9 & 6 & $1.4(-11)$ & $2.3(-7)$ & $3.6(-7)$ \\
\hline 2000 & 98 & 17 & 12 & $1.4(-11)$ & $4.5(-7)$ & $7.3(-7)$ \\
\hline 3000 & 165 & 26 & 19 & $1.4(-11)$ & $6.8(-7)$ & $1.1(-6)$ \\
\hline 4000 & 199 & 35 & 25 & $1.6(-11)$ & $1.0(-6)$ & $1.5(-6)$ \\
\hline \multicolumn{7}{|c|}{ 2D NOCl model } \\
\hline 500 & 38 & 81 & 75 & & & \\
\hline 1000 & 63 & 145 & 128 & & & \\
\hline 1500 & 98 & 220 & 211 & & & \\
\hline
\end{tabular}

${ }^{\mathrm{a}} \mathrm{CPUT}=\mathrm{CPU}$ time (second).

${ }^{\mathrm{b}}$ Error $=1 / N \Sigma_{i=1}^{N}\left|\exp \left\{R\left(\mathbf{r}_{i}, t\right)^{\text {exact }}\right\}-\exp \left\{R\left(\mathbf{r}_{i}, t\right)^{\text {calcu }}\right\}\right|$.

${ }^{\mathrm{c}}$ Calculations done on a Sun workstation OS 5.6.

${ }^{\mathrm{d}}$ Calculations done on Cray J90 at Lawrence Berkeley National Laboratory. SO calculations were not per-

formed on the $3 \mathrm{D}$ free particle model due to large CPU time consumption.

QFD method only to $57^{2}$. The ratio of the former to latter is $\sim 1300$ times in favor of the QFD. The storage needed for the QFD method scales roughly as $n^{2}$, the size of the local interpolation matrix, with $n$ slowly growing with the dimension of the system (see Table II).

In the case of 2D and 3D free particles, despite using a short-time second-order propagator (like the SO method), the QFD method is comparable in time efficiency to the CE and SO methods in the 2D case and is far superior in the $3 \mathrm{D}$ case. The CPU time for the QFD method scales approximately as $N_{n} n^{3} \times$ total propagation time/ $\Delta t$, where $N_{n}$ is defined in step 7 of Sec. III B. As discussed above, the growth of $n$ with the dimension of the system is generally slow. Table III shows the comparison of CPU time. The data for the 3D free particle was obtained on a CRAY J90 supercomputer. In addition, no data entries are presented for the SO method in the $3 \mathrm{D}$ free particle case because it requires almost three times more of CPU time than the CE method.

The 2D coherent-state model can pose a challenge to the Lagrangian moving grid QFD method. The harmonic potential of the 2D coherent-state model reaches its maximum on the truncated boundary, and the corresponding density $\rho$ with energy below the cutoff must always preserve its shape when it propagates to and fro and evolves into complicated quantum interference patterns. The QFD method must be able to guide the associated moving grids to constantly adjust their trajectories. Even if only one moving grid point, particularly one that carries significant density value, becomes out of step with the rest of grid points at any instant of time, then the coherent-state wave function will collapse soon afterward. Table III shows that the QFD method can yield excellent results for the 2D coherent state, on par with the SO method in both accuracy and efficiency, while the CE method is more accurate but less efficient. Here, no absorbing potentials were implemented in the CE and SO methods, and to avoid unphysical boundary reflection, proper regions have been optimally chosen for different lengths of propagation
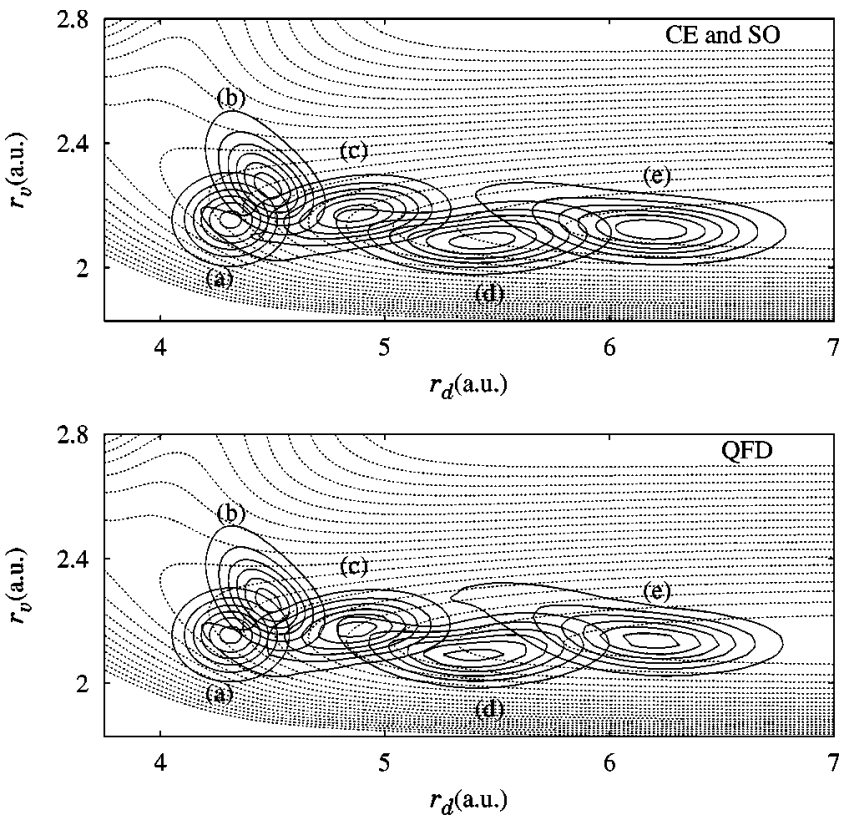

FIG. 1. Contour snaphots of the density $\rho$ for the 2D NOCl photodissociation model superimposed on its $S_{1}$ potential energy surface contours as a function of the Jacobi coordinates $r_{d}$ and $r_{v}$ for fixed angle $\theta_{0}=127.4$ at five instants of time: (a) $t=0$ a.u. (b) $t=500$ a.u., (c) $t=1000$ a.u., (d) $t=1500$ a.u., (e) $t=2000$ a.u.. The upper panel corresponds to the results of the CE and SO methods and the lower one to the QFD method. 
time.

It is especially instructive to note that, in the $2 \mathrm{D}$ and $3 \mathrm{D}$ free particle cases and for long time propagation, the QFD method is more favorable in terms of accuracy when compared to the CE and SO methods. This can be explained as follows. As the propagation proceeds, the wave packet disperses rapidly and its real and imaginary parts become more and more oscillatory with the growth of its phase [see Eq. (4.2)]. As a result, the initial number of fixed Fourier grid points eventually becomes insufficient to give an accurate spatial representation. On the other hand, in the Lagrangian QFD method, not only do the quantities $R$ and $S$ stay smooth, but the underlying grid points also move with the wave packet, thus, maintaining the quality of spatial interpolation throughout the evolution.

To further examine the Lagrangian QFD method, snapshots of the density contours for the $2 \mathrm{D} \mathrm{NOCl}$ photodissociation model at five different instants of time are displayed in Fig. 1. The upper panel gives the results of the CE and SO methods and the lower panel those of the QFD method. It can be seen that these results are in excellent agreement with each other and also with the results of Manthe et al. [60] and Untch et al. [61]. The same $\mathrm{NOCl}$ photodissociation process with similar snapshots can be found in these two papers.

\section{SUMMARY}

The following points and conclusions can be drawn from the present study. (1) The first implementation of QFD is presented within the Lagrangian description using the quantities $R$ and $S$, instead of $\rho$ and $\mathbf{v}$; (2) the smoother and simpler structure of $R$ is exploited to further raise the computational efficiency and accuracy, as compared to those using $\rho$; (3) radial basis functions (or reproducing kernels) are suitable for the spatial representation of moving grids and are capable of yielding accurate interpolation of the smooth field quantities $R$ and $S$ and their first and second derivatives; (4) the Lagrangian version of the QFD method does not suffer from boundary reflection of the evolving wave packet and hence no artificial absorbing potentials are needed, in contrast to solving the Schrödinger equation using a fixed grid; and (5) the particle or trajectory picture of a quantum system can be obtained by interpolating moving grids.

The numerical development of the QFD method is in its infancy, and there is ample room for further improvement. A central task in the Lagrangian QFD method is the establishment of a stable and efficient spatial representation, e.g., the radial basis functions, for the moving grid interpolations. Higher-order time integrators can also be introduced by using various sophisticated integration procedures, e.g., RungeKutta schemes. It is also important to develop a proper means to analyze the data available from the Lagrangian QFD method because of the irregularity of the grids. Finally, further applications of the QFD method to realistic boundstate problems would help settle open questions regarding the node issue [9].

\section{ACKNOWLEDGMENT}

The authors acknowledge support from the Department of Energy.
[1] C. Leforestier, R. H. Bisseling, C. Cerjan, M. D. Feit, R. Friesner, A. Guldberg, A. Hammerich, G. Jolicard, W. Karrlein, H. D. Meyer, N. Lipkin, O. Roncero, and R. Kosloff, J. Comput. Phys. 94, 59 (1991).

[2] Time-Dependent Quantum Molecular Dynamics, edited by J. Broeckhove and L. Lathouwers, (Plenum Press, New York, 1992).

[3] R. Kosloff, Annu. Rev. Phys. Chem. 45, 145 (1994).

[4] E. Deumens, A. Diz, R. Longo, and Y. Öhrn, Rev. Mod. Phys. 66, 917 (1994).

[5] B. Jackson, Annu. Rev. Phys. Chem. 46, 251 (1995).

[6] N. Balakrishnan, C. Kalyanaraman, and N. Sathyamurthy, Phys. Rep. 280, 79 (1997).

[7] S. Goldstein, Phys. Today 51 (3), 42 (1998); 51 (4), 38 (1998).

[8] E. Deotto and G. C. Ghirardi, Found. Phys. 28, 1 (1998).

[9] P. R. Holland, The Quantum Theory of Motion (Cambridge University Press, Cambridge, England, 1993).

[10] D. Bohm and B.J. Hiley, The Undivided Universe: An Ontological Interpretation of Quantum Theory (Routledge, London, 1993).

[11] K. Berndl, D. Dürr, S. Goldstein, G. Peruzzi, and N. Zanghi, Commun. Math. Phys. 173, 647 (1995).

[12] E. Madelung, Z. Phys. 40, 322 (1926).

[13] L. de Broglie, C. R. Acad. Sci. Paris 183, 447 (1926); 184, 273 (1927).

[14] D. Bohm, Phys. Rev. 85, 166 (1952); 85, 185 (1952).

[15] P. M. Gerhart, R. J. Gross, and J. I. Hochstein, Fundamentals of Fluid Mechanics, 2nd ed. (Addison-Wesley, New York, 1992).

[16] J. H. Weiner and A. Askar, J. Chem. Phys. 54, 1108 (1971); 54, 3534 (1971).

[17] A. Askar and J. H. Weiner, Am. J. Phys. 39, 1230 (1971).

[18] G. Terleck, N. Grün, and W. Scheid, Phys. Lett. 88A, 33 (1982); J. Phys. B 17, 3719 (1984).

[19] P. Zimmerer, M. Zimmermann, N. Grün, and W. Scheid, Comput. Phys. Commun. 63, 21 (1991).

[20] F. S. Mayor, A. Askar, and H. A. Rabitz, J. Chem. Phys. 11, 2423 (1999).

[21] C. L. Lopreore and R. E. Wyatt, Phys. Rev. Lett. 82, 5190 (1999).

[22] R. E. Wyatt, J. Chem. Phys. 111, 4406 (1999).

[23] A. S. Bamzai and B. M. Deb, Rev. Mod. Phys. 53, 95 (1981); 53, 593 (1981)

[24] S. K. Ghosh and B. M. Deb, Phys. Rep. 92, 1 (1982); correction 53, 593 (1981).

[25] B. M. Deb and S. K. Ghosh, J. Chem. Phys. 77, 342 (1982).

[26] M. Himi and K. Fukushima, Nucl. Phys. A 431, 161 (1984).

[27] P. K. Chattaraj, S. Rao Koneru, and B. M. Deb, J. Comput. Phys. 72, 504 (1987).

[28] B. M. Deb and P. K. Chattaraj, Phys. Rev. A 37, 4030 (1988); 39, 1696 (1989); Chem. Phys. Lett. 148, 550 (1988).

[29] C. R. Leavens, G. Iannaccone, and W. R. Mckinnon, Phys. Lett. A 208, 17 (1995). 
[30] B. K. Dey and B. M. Deb, Int. J. Quantum Chem. 67, 251 (1998); 56, 707 (1995).

[31] P. K. Chattaraj and S. Sengupta, J. Phys. Chem. 100, 16126 (1996); Phys. Lett. A 181, 225 (1993).

[32] Z. X. Chen, B. Cockburn, C. L. Gardner, and J. W. Jerome, J. Comput. Phys. 117, 274 (1995).

[33] U. Schwengelbeck and F. H. M. Faisal, Phys. Lett. A 199, 281 (1995); 207, 31 (1995).

[34] S. Ohnishi, Sci. Rep. Res. Inst. Tohoku Univ. A 41, 157 (1996).

[35] B. K. Dey, A. Askar, and H. A. Rabitz, J. Chem. Phys. 109, 8770 (1999).

[36] X.-G. Hu, T.-S. Ho, and H. Rabitz, Chem. Phys. Lett. 288, 719 (1998); Comput. Phys. Commun. 113, 168 (1998); Phys. Rev. E 61, 2074 (2000).

[37] W. H. Press, S. A. Teukolsky, W. T. Vetterling, and B. P. Flannery, Numerical Recipes in $C$, 2nd ed. (Cambridge University Press, Cambridge, 1992).

[38] J. C. Light, I. P. Hamilton, and J. V. Lill, J. Chem. Phys. 82, 1400 (1985).

[39] D. Gottlieb and S. A. Orszag, Numerical Analysis of Spectral Methods: Theory and Applications (SIAM, Philadelphiia, 1983).

[40] R. Kosloff, in Dynamics of Molecules and Chemical Reactions, edited by R. E. Wyatt and J. Z. H. Zhang (Marcel Dekker, New York, 1996).

[41] D. K. Hoffman, T. L. Marchioro, II, M. Arnold, Y. Huang, W. Zhu, and D. J. Kouri, J. Math. Chem. 20, 117 (1994).

[42] N. J. D. Powell, in Advances in Numerical Analysis II: Wavelets, Subdivision Algorithms, and Radial Basis Functions, edited by W. A. Light (Oxford University Press, Oxford, 1992), p. 105.
[43] R. Schaback, in Approximation Theory VIII: Approximation and Interpolation, edited by C. K. Chui and L. L. Schumaker (World Scientific, Singapore, 1995), Vol. 1, p. 491.

[44] C. A. Micchelli, Constr. Approx. 2, 11 (1986).

[45] R. Schaback, Ann. Numer. Math. 4, 547 (1997).

[46] R. Schaback, in Multivariate Approximations: From CAGD to Wavelets, edited by K. Jetter and F. Utreras (World Scientific, Singapore, 1993), p. 293.

[47] N. Aronszajn, Trans. Am. Math. Soc. 68, 337 (1950).

[48] T. Hollebeek, T.-S. Ho, and H. Rabitz, Annu. Rev. Phys. Chem. 50, 537 (1999).

[49] N. Dyn, in Approximation Theory VI, edited by C. K. Chui, L. L. Schumaker, and J. D. Ward (Academic Press, New York, 1989), Vol. 1, p. 211.

[50] E. J. Kansa, Comput. Math. Appl. 19, 127 (1990); 19, 147 (1990).

[51] B. W. Shore, J. Chem. Phys. 59, 6450 (1973).

[52] M. J. Davis and E. J. Heller, J. Chem. Phys. 71, 3383 (1979).

[53] I. P. Hamilton and J. C. Light, J. Chem. Phys. 84, 306 (1986).

[54] W. Yang and A. C. Peet, Chem. Phys. Lett. 153, 98 (1988).

[55] Z. Bačić and J. C. Light, Annu. Rev. Phys. Chem. 40, 469 (1989).

[56] M. D. Feit, J. A. Fleck, Jr., and A. Steiger, J. Comput. Phys. 47, 412 (1982).

[57] A. Askar and A. S. Cakmak, J. Chem. Phys. 68, 2794 (1978).

[58] J. K. McDonald, J. A. Merrit, V. F. Kalasinksky, H. L. Heusel, and J. R. During, J. Mol. Spectrosc. 117, 69 (1986).

[59] R. Schinke, M. Nonella, H. U. Suter, and J. R. Huber, J. Chem. Phys. 93, 1098 (1990).

[60] U. Manthe, H. D. Meyer, and L. S. Cederbaum, J. Chem. Phys. 97, 3199 (1992).

[61] A. Untch, K. Weide, and R. Schnike, J. Chem. Phys. 95, 6496 (1991). 